\title{
Novel Biomarkers in Cholangiocarcinoma
}

\author{
Dan G. Duda ${ }^{1,{ }^{*}}$, Shuichi Aoki ${ }^{1, *}$, Simona Dima ${ }^{2}$, Irinel Popescu' ${ }^{2}$, Mitesh J. Borad ${ }^{3}$
}

'Edwin L. Steele Laboratories for Tumor Biology, Department of Radiation Oncology, Massachusetts General Hospital, Boston, USA

${ }^{2}$ Center of Digestive Diseases and Liver Transplantation, Fundeni Clinical Institute, Bucharest, Romania

${ }^{3}$ Divison of Hematology/Oncology, Mayo Clinic, Phoenix, USA

\author{
Corresponding author: \\ Dan G. Duda, DMD, PhD \\ Department of Radiation Oncology \\ Massachusetts General Hospital \\ Cox-7, 100 Blossom Street \\ Boston, MA 02114, USA \\ Tel: 1-617-726-4648 \\ Fax: 1-617-726-1962. \\ E-mail: duda@steele.mgh.harvard.edu \\ *These authors contributed equally to \\ this work.
}

\section{INTRODUCTION}

Cholangiocarcinoma (CCA) is the second most common type of primary liver cancer, after hepatocellular carcinoma, and accounts for $10-25 \%$ of primary liver cancers $(1,2)$. CCAs are classified as intrahepatic CCA (iCCA) and extrahepatic CCA (eCCA), according to their anatomical location (3). CCA is more frequent in South Asia compared to Western countries, because of the increased prevalence of established risk factors, including fluke infections (2). In the USA, the incidence of CCA has increased over the past three decades (4). Surgical resection and transplantation are the only curative treatments for patients with CCA, however the majority of cases are not surgical candidates when diagnosed (5). Even after potentially curative surgery, the prognosis of patients with CCA remains poor with 5 year-survival rates of $20 \%$ (6). Gemcitabine in combination with cisplatin is widely accepted as a frontline standard therapy for advanced or metastatic CCA (7). However, beyond these treatments, there are limited effective systemic therapy options.

Recently, an array of actionable genetic alterations has been identified in CCA (8). Studies evaluating these biomarkers could provide insights into the mechanisms of CCA tumorigenesis and identify potential targets for therapy (9). Genome-wide characterization have highlighted differing molecular aberrations of CCA and they could help to stratify CCA patients for molecularly targeted therapies (10). Previous studies have investigated roles of genetic, epigenetic and transcriptomic alterations along with well characterized paradigms such as oncogene and tumor-suppressor alterations. Additionally, the comparison between CCA and benign disease has been useful in identifying differences in carcinogenic mechanisms (11). These studies identified genetic alterations in CCA that could potentially lead to early diagnosis and precision treatment. This review article discusses the potential therapeutic biomarkers in CCA and the steps for their clinical translation. 


\section{INTEGRATED WHOLE-GENETIC PROFILING ANALYSIS FOR CCA}

We have performed profiling studies of the genomic and transcriptomic landscapes of each CCA subtype, in an attempt to clarify their distinct biological behavior and use the information of patients' stratification for molecularly targeted therapies (10). In Jusakul et al., we proposed four main subtypes of CCA based on integrative clustering of mutation, copy number, gene expression, and epigenetic data from nearly 500 CCA samples (9). Fluke-positive CCAs, which were classified as cluster 1 and 2, are enriched in ERBB2 amplifications and TP53 mutations. Conversely, fluke-negative CCAs exhibit high copy-number alterations and PD-1/PD-L2 expression (cluster 3), or epigenetic mutations (IDH1/2, BAP1) and FGFR/PRKA-related gene rearrangements (cluster 4). The cluster 1 or 2 of CCAs are characterized as elevated mutation rates and increased point-mutation sub-clonality, compared with other clusters, especially cluster 4 CCAs. Cluster 1 or 2 CCAs are driven by external carcinogenic agents and early epigenetic deregulation so that the processes of carcinogen-induced methylation, deamination, and mutation are inherently stochastic from cell to cell and lead to increased levels of intratumor heterogeneity. On the other hand, cluster 4 CCAs are likely driven by genetic events such as $I D H 1 / 2$ or BAPI mutations, with epigenetic aberrations arising as a downstream consequence. Therefore, dominant and critical somatic mutations (IDH1/2 and BAP1) may occur as a primary event and drive both rapid clonal outgrowth, which directly induces DNA hypermethylation. Specifically, IDH1/2 mutations have been shown to increase 2-hydroxyglutarate oncometabolite production, leading to DNA hypermethylation (12).

These signature genomic alterations in each subtype highlights potential therapeutic opportunities. For example, it is possible that CCAs in clusters 1 or 2 with ERBB2 amplification may be appropriate for therapies targeting ERBB2 (HER2) signaling (13). In cluster 4, FGFR fusion rearrangements and $I D H 1 / 2$ mutations are the most promising targeted treatments (14). The elevated expression of immune related genes in cluster 3 CCAs suggests a therapeutic opportunity for immunotherapy.

Another whole-exome and transcriptome sequencing in a large cohort of 260 CCA patients proposed that nearly $40 \%$ of CCA patients obtains genetic alterations for potential therapeutic targets (15). In this study, recurrent mutations in IDH1, IDH2, FGFR1, FGFR2, FGFR3, EPHA2, and $B A P 1$ were identified predominantly in iCCA, whereas ARID1B, ELF3, PBRM1, PRKACA, and PRKACB mutations/alterations occurred preferentially in eCCA.
Notably, other reports have identified that FGFR2 fusions result in ligand-independent activation of this receptortyrosine kinase exclusively in patients with iCCA (16-20). Novel gene fusions involving PRKACA or PRKACB, were discovered only in eCCA and these aberrations induced by gene fusions are considered potentially druggable. This study also reported that the subgroup with the poorest prognosis had significant enrichment of hypermutated tumors and a characteristic elevation in the expression of immune checkpoint molecules. Since the total number of somatic mutations (tumor mutation burden) are associated with clinical response to immune checkpoint blockade therapies in melanoma and lung cancer $(21,22)$, immune-modulating therapies might also be potentially promising options for this tumor subtype.

Sia et al. presented two subtypes of CCA, based on integrative genomic analysis using formalin-fixed CCA samples from 149 patients (23). One was represented by the proliferation type, which is characterized by activation of molecular characteristics such as stem-cell like iCCA, oncogenic pathways (IGF1R, MET, EGFR), and oncogenic mutations (KRAS, BRAF and EGFR). This type is associated with a poor prognosis, consistent with prior reports (24). The other was the inflammationrelated type, which is associated with activation of inflammatory signaling, overexpression of chemokine and cytokines such as IL-6, IL-10 and IL-17, and STAT3 constitutive activation. The overexpression mediators of Th2 immune responses, such as IL-4 and IL-10, downregulation of Th1 cytokines, and STAT3 activation, a key transducer of cytokine signaling (25), suggest a potential role for immunotherapy targeting immunosuppressive factor expression by cancer cells.

Zhong et al. identified candidate biomarkers with high potential for clinical application in CCA using a meta-analysis-based approach (26). They collected the integrated analysis of 7 eligible datasets $(9,11,23,24$, 27-29), which included a total of 428 cases and 46 controls, and identified a set of 1,080 differentially expressed genes, including 710 upregulated and 370 downregulated genes. The most upregulated genes associated with CCA were SPP1, MMP11, COL1A1, TMSB10, AGRN, and COL4A1 (30-34). By evaluating the functional mechanism using gene ontology (GO) and Kyoto Encyclopedia of Gene and Genomes (KEGG) pathway enrichment analysis, the most significantly enriched terms are associated with activated cell cycle process and extracellular components. CCA has abundant of stroma and the genes of SPP1, MMP11, COL1A1 may regulate the production and remodeling of stromal elements (35). This characteristic CCA 
microenvironment may be a crucial component governing cancer progression and treatment response, and might represent an important target for new therapy approaches in CCA (36).

\section{EMERGING MOLECULAR TARGETED THERAPIES}

\section{IDH1/2 as potential therapeutic targets in $i C C A$}

Mutation in IDH genes in iCCA leads to abnormal enzymatic activity allowing them to convert a-ketoglutarate (aKG) to 2-hydroxyglutarate (2HG). This oncometabolite can inhibit the activity of multiple aKG-dependent dioxygenases, which results in alterations in cell metabolism, differentiation, survival, and extracellular matrix maturation (37-41). The oncogenic effects of $I D H$ mutations appear to be primarily mediated through epigenetic alterations. The reduction of aKG production and reduced activity of aKGdependent enzymes in IDH mutant cancers alters epigenetic states, which means histone and DNA hypermethylation. $I D H 1$ and $I D H 2$ mutations are detected predominantly in iCCAs, ranging from $18 \%$ to $36 \%$ of cases $(18,42-44)$. The vast majority of $I D H 1$ and $I D H 2$ mutations are novel substitution mutations recently targeted for molecule inhibition.

Since small-molecule inhibitors of mutant $I D H 1$ or $I D H 2$ have shown favorite efficacy in leukemia $(45,46)$, orally bioavailable inhibitors (AG120) have also moved to clinical trials for patients with $I D H 1$ mutationsharboring CCA. Preliminary results from a phase I trial of AG-120 have shown an acceptable safety profile in dose-escalation and dose-expansion cohorts. Moreover, among 20 patients with CCA treated with AG-120, one had a partial response and 11 showed disease stabilization. A multicenter, double-blind, placebo-controlled phase III trial in 186 patients with IDH1-mutant CCA is currently ongoing (NCT02989857). On the other hand, enasidenib, an oral selective inhibitor of mutant $I D H 2$, has shown activity against acute myeloid leukemia (4749). Enasidenib is also currently being evaluated in a multicenter phase $\mathrm{I} / \mathrm{II}$ trial in patients with $\mathrm{IDH}$-mutant advanced cancers, including iCCA (NCTO2273739) (table 1).

\section{FGFR2 as a potential therapeutic target in $C C A$}

FGFR2 is another attractive target in iCCAs. FGFR2 alterations are frequently found (in $13-20 \%$ of iCCAs)
$(17,42)$. FGFR2 fusions represent a majority of FGFR2 arrangement in CCA and are associated with longer survival compared with CCA without FGFR2 translocation (17). As discussed above, FGFR2 translocations have been identified as a potential target for tyrosine kinase inhibitor therapies.

The pan-FGFR inhibitor NVP-BGJ398 has shown potential anti-tumor efficacy in preclinical models of CCA (50), and a phase II study in patients with advanced CCA with FGFR alterations is ongoing (NCT02150967) (table 1). Preliminary data from this study showed that NVP-BGJ398 may indeed have promising anti-tumor activity, with a disease-control rate of $82 \%$ and a manageable safety profile (51). Erdafitinib, which is another orally pan-FGFR inhibitor (52), is currently being tested in a phase II trial (NCT02699606). In a previous phase I dose-escalation study (NCT01703481) (table 1), erdafitinib showed a manageable safety profile at the doses tested and favorable clinical responses. Among the 23 patients with solid tumors harboring FGFR alterations enrolled in this trial, four patients had a confirmed response to erdafitinib treatment, one had an unconfirmed partial response and 16 had stable disease (53). Other FGFR-selective inhibitors are currently evaluated in patients with advanced solid-organ malignancies, for example ARQ 087 (NCT03230318), TAS-120 (NCT02052778) and INCB054828 (NCT02924376, NCT02393248) (table 1). Finally, ponatinib, a multikinase inhibitor with anti-FGFR activity, has also shown promising efficacy in patients with advanced iCCA with FGFR2 fusions (16), and is currently being evaluated in a phase II trial (NCT02265341) (table 1).

\section{KRAS and BRAF mutations as a target in $C C A$}

Activating mutations in the KRAS gene are oncogenic driver mutations that frequently occur in CCA (11$25 \%$ of cases) $(15,18,42)$. KRAS activation upregulates downstream pathways including the RAF/MEK/ERK (MAPK) pathway and is associated with poor survival in CCA patients $(18,44,54)$. Phase Ib and II studies of selumetinib, a selective MEK inhibitor, demonstrated median PFS rates of 6.4 and 3.7 months, respectively, in advanced CCA patients $(55,56)$. BRAF mutations also occur in some CCAs (3-5\% of cases) $(44,54,57)$ (table 1). The most common activating mutations are V600E BRAF mutations. In melanoma patients with V600E BRAF mutations, BRAF inhibition leads to robust responses in a majority of patients. Among eight CCA patients with BRAF mutation, treatment with the oral 
Table 1 - The selected biomarker and clinical trials in cholangiocarcinoma

\begin{tabular}{|c|c|c|c|c|c|}
\hline Biomarker & Pathway & Incidence (\%) & Agents & Clinical trial & Phase \\
\hline FGFR2 fusion & FGFR & 13-20 (iCCA) & $\begin{array}{l}\text { ARQ } 087 \\
\text { BGJ398 } \\
\text { Ponatinib } \\
\text { INCB054828 } \\
\text { Dovitinib, gemcitabine and capecitabine } \\
\text { H3B-6527 } \\
\text { Erdafitinib } \\
\text { TAS-120 }\end{array}$ & $\begin{array}{l}\text { NCT03230318 } \\
\text { NCT02150967 } \\
\text { NCT02265341 } \\
\text { NCT02924376 } \\
\text { NCT01497392 } \\
\text { NCT02834780 } \\
\text { NCT02699606 } \\
\text { NCT02052778 }\end{array}$ & $\begin{array}{l}\text { II } \\
\text { II } \\
\text { II } \\
\text { II } \\
\text { I } \\
\text { I } \\
\text { II } \\
1 / / I\end{array}$ \\
\hline IDH1/2 mutation & Krebs cycle & 18-36 (iCCA) & $\begin{array}{l}\text { AG-120 } \\
\text { AG-221 } \\
\text { IDH305 }\end{array}$ & $\begin{array}{l}\text { NCT02989857 } \\
\text { NCT02273739 } \\
\text { NCT02381886 }\end{array}$ & $\begin{array}{l}\text { III } \\
1 / I 1 \\
I\end{array}$ \\
\hline BRAF mutation & RAF/MEK/ERK & $3-5$ (iCCA) & $\begin{array}{l}\text { Vemurafenib } \\
\text { Dabrafenib and trametinib } \\
\text { Gemcitabine, cisplatin and MEK162 }\end{array}$ & $\begin{array}{l}\text { NCT02304809 } \\
\text { NCT02034110 } \\
\text { NCT01828034 }\end{array}$ & $\begin{array}{l}\text { II } \\
\text { II } \\
\text { I/II }\end{array}$ \\
\hline $\begin{array}{l}\text { MET overexpression } \\
\text { MET amplification }\end{array}$ & MET & $\begin{array}{l}12 \text { (iCCA), } 16 \text { (eCCA) } \\
2-7 \text { (iCCA, eCCA) }\end{array}$ & $\begin{array}{l}\text { Cabozantinib } \\
\text { Merestinib }\end{array}$ & $\begin{array}{l}\text { NCT01954745 } \\
\text { NCT03027284 }\end{array}$ & II \\
\hline $\begin{array}{l}\text { EGFR overexpression } \\
\text { EGFR mutation }\end{array}$ & EGFR & $\begin{array}{l}100 \text { (iCCA), } 52.6 \text { (eCCA) } \\
0-15 \text { (iCCA), } 15 \text { (eCCA) }\end{array}$ & $\begin{array}{l}\text { Erlotinib } \\
\text { Panitumumab + gemcitabine/irinotecan }\end{array}$ & $\begin{array}{l}\text { NCT00033462 } \\
\text { NCT00948935 }\end{array}$ & II \\
\hline DKK-1 & Wnt/b catenin & 38.5 (iCCA) & DKN-01 + gemcitabine/cisplatin & NCT02375880 & I \\
\hline BAP1 mutation & Deubiquitinase & 9-26 (iCCA), 5-10 (eCCA) & Tazemetostat & None & $\begin{array}{l}\text { Pre- } \\
\text { clinical }\end{array}$ \\
\hline $\begin{array}{l}\text { PI3CA } \\
\text { amplification/mutation } \\
\text { Notch }\end{array}$ & $\begin{array}{l}\mathrm{PISK/AKT/mTOR} \\
\text { Notch }\end{array}$ & $\begin{array}{l}25.5 \text { (iCCA), } 40 \text { (eCCA) } \\
17-65 \text { (iCCA) 6-56 (eCCA) }\end{array}$ & $\begin{array}{l}\text { Everolimus } \\
\text { Copanlisib+gemcitabine/cisplatin } \\
\text { LY3039478+other anticancer agents }\end{array}$ & $\begin{array}{l}\text { NCT00973713 } \\
\text { NCT02631590 } \\
\text { NCT02784795 }\end{array}$ & $\begin{array}{l}\text { II } \\
\text { II } \\
\text { I }\end{array}$ \\
\hline PARP & PARP & & Niraparib & NCT03207347 & II \\
\hline $\begin{array}{l}\text { HER2 } \\
\text { amplification/overexpression }\end{array}$ & ERBB2 & 1 (iCCA), 5-9 (eCCA) & $\begin{array}{l}\text { Trastuzumab } \\
\text { Lapatinib } \\
\text { Pertuzumab } \\
\text { ASLAN001 }\end{array}$ & $\begin{array}{l}\text { NCT00478140 } \\
\text { None } \\
\text { None } \\
\text { NCT02609958 }\end{array}$ & $\begin{array}{l}\text { II } \\
\text { Case } \\
\text { series } \\
\text { Case } \\
\text { series } \\
\text { II }\end{array}$ \\
\hline VEGFR2 & & 60 (iCCA) & $\begin{array}{l}\text { Ramucirumab } \\
\text { Apatinib }\end{array}$ & $\begin{array}{l}\text { NCT02520141 } \\
\text { NCT03521219 }\end{array}$ & II \\
\hline VEGFR/PDGFR/RAF & & & $\begin{array}{l}\text { Pazopanib+GSK1120212 (MEK inhibitor) } \\
\text { Pazopanib+gemcitabine }\end{array}$ & $\begin{array}{l}\text { NCT01438554 } \\
\text { NCT01855724 }\end{array}$ & II \\
\hline EGFR/VEGFR/PDGFR/RAS/RA & & & Regorafenib & NCT02053376 & II \\
\hline TrkA/B/C/ALK/ROS1 & & & Entrectinib & NCT02568267 & "I \\
\hline
\end{tabular}

iCCA, intrahepatic CCA: eCCA, extrahepatic CCA

BRAF inhibitor vemurafenib led to a partial response in one patient (58).

\section{MET as a target in CCA}

Signaling of hepatocyte growth factor (HGF) through its tyrosine kinase receptor MET is important in cellular processes mediating survival and invasion and is thought to be a key mediator of cancer metastasis (23). Previous studies have indicated that MET is commonly expressed in both iCCA (20-58\% of cases) and eCCA (0-68\% of cases) (59-61), and that iCCA cell proliferation is characterized by activation of MET, EGFR, and MAPK signaling (23). However, early phase clinical trials of MET inhibitors in patients with CCA have failed to show significant activity. A phase I study (62) of the MET inhibitor tivantinib in combination with gemcitabine in patients with CCA exhibited partial responses and stable disease in $20 \%$ and $46 \%$ of patients, respectively (table 1). Cabozantinib, a multikinase inhibitor against MET and VEGFR2, had shown a median PFS of 1.8 months with limited activity and substantial toxicity in unselected CCA patients (63). Moreover, plasma soluble MET, not tissue MET expression, correlated with patient outcome in this study. It remains to be unclear whether tissue or plasma soluble MET could be useful biomarkers for the selection of patients for treatment using MET inhibitors. 


\section{EGFR as a target in CCA}

Increased EGFR expression has been associated with poor prognosis, and there is a subpopulation with significant enrichment related to EGFR signaling based on genomic analyses in CCA patients (24). EGFR activation promotes cell proliferation, migration and tumor angiogenesis. EGFR expression was identified in 38$100 \%$ of CCA patients (64). These analyses suggested that anti-EGFR treatment might benefit certain CCA subpopulations. However the results of early phase clinical trial of EGFR inhibitors have been disappointing. A phase II trial of EGFR inhibitor erlotinib in CCA patients showed an overall response rate (ORR) of $8 \%$ and PFS of $17 \%$ at 6 months and was terminated early (65) (table 1). Thus, EGFR expression by IHC is not necessarily associated with treatment response to EGFR inhibitors and has not been a reliable biomarker thus far.

\section{WNT/DKK1 as a target in CCA}

Dickkopf-1 (Dkk1) is an antagonist of Wht signaling that is overexpressed in many cancers, including CCA, and has been demonstrated to promote cell migration, tumor growth and angiogenesis. The positive expression of Dkk1 was identified in $38.5 \%$ of iCCA and considered an unfavorable predictor for overall survival (66). The oncogenic mechanism of action of Dkk1 occurs in part through the activation of non-canonical Wnt signaling and PI3K/Akt signaling in cancer cells. In addition, recent studies have demonstrated that Dkk1 has immune modulatory activity that promotes tumorigenesis (67). Dkk1 contributes to an immunosuppressive tumor microenvironment in syngeneic lung and melanoma models by inhibiting canonical Wnt signaling in myeloid-derived suppressor cells (MDSCs) (68). Neutralization of Dkk1 blocked MDSC activity and results in tumor regression. Furthermore, Dkk1 also promotes immune evasion of metastatic cancer cells by downregulating NK activating ligands (69). Inhibiting Dkk1 expression results in NK recognition and eradication of these cells. Moreover, in CCA patients, anti-Dkk1 antibodies (DKN-01) showed greater than expected efficacy in recent phase I trials (NCT02375880) (table 1).

\section{IMMUNOTHERAPY IN CCA}

Immunotherapy using immune checkpoint blockade (ICB) has shown great promise in oncology, but its impact in $\mathrm{Gl}$ cancers has been more limited so far, including in CCA. Among many candidate biomarkers of a response
ICB, the most investigated candidate has been expression of the PD-1 ligand, PD-L1. The expression of PD-L1 on tumor cells has shown inconsistent correlations with response to ICB monotherapy in melanoma and nonsmall-cell lung cancer (NSCLC). In CCA, recent reports have shown widely variable PD-L1 expression $(9-72 \%$ of specimens) and immune cell infiltration within the CCAs (46-63\%) $(70,71)$. However, similar to pancreatic cancer, a substantial proportion of CCAs have a highly immunosuppressive tumor-associated stroma, consisting of activated cancer-associated fibroblasts, endothelial cells, MDSCs and tumor-associated macrophages. This characteristic CCA microenvironment may modulate anticancer immune responses and impair the efficacy of ICB treatment. Further investigation of PD-L1 expression and the immunosuppressive cues in CCA microenvironment are warranted to clarify their roles as biomarkers of response or resistance to ICB therapies.

The presence of tumor DNA mismatch repair (MMR) deficiency and/or microsatellite instability (MSI) is associated with high rates and durability of responses to ICB therapy across multiple tumor types since the accumulation of genetic aberrations might relate to the expression of neoantigens capable of eliciting an antitumor T-cell response (72-74). Indeed, MMR deficiency was detected in $5-10 \%$ of CCA (75). Moreover, the cumulative mutational burden in tumor has been proposed as a biomarker for responsiveness to ICB in melanoma and $\operatorname{NSCLC}(21,22)$. According to a wholeexome-sequencing study of 231 CCA tumor samples (15), a median of 39 and 35 somatic nonsynonymous mutations were identified in iCCA and eCCA, respectively. Nearly $6 \%$ of CCAs show hypermutated status and $36 \%$ of these hypermutated tumors has concurrent MMR deficiency and/or MSI. On the other hand, the median number of non-synonymous mutations was 302 in patients with NSCLC who are considered to obtain durable clinical benefit from pembrolizumab (21). These data suggest that ICB and immunemodulating therapies are possibly promising, especially for the subgroup of patients with CCA with high mutational loads.

Indeed, many studies are now testing the use of ICBs in CCA patients (see table 2). The KEYNOTE-028 study is testing pembrolizumab to CCAs harboring $\geq 1 \%$ PD-L1 expression in the tumor. The ORR was $17 \%$ and the disease control rate was $34 \%$. There were also a number of durable responders (22\%) that continued to benefit from pembrolizumab over 40 weeks. This promising safety and efficacy of pembrolizumab in the KEYNOTE-028 prompted the next enrollment for 100 CCAs patients in the ongoing KEYNOTE-158 trial 
Table 2 - Clinical trials of immunotherapy for cholangiocarcinoma

\begin{tabular}{|c|c|c|c|}
\hline \multicolumn{2}{|l|}{ Proposed therapy } & Phase & Clinical trial \\
\hline \multicolumn{4}{|l|}{ Single ICB } \\
\hline & Pembrolizumab (anti-PD-1 antibody) & $\|$ & NCT03110328 \\
\hline & Pembrolizumab (anti-PD-1 antibody) & II & NCT02628067 \\
\hline & Nivolumab (anti-PD-1 antibody) & II & NCT02829918 \\
\hline & Durvalumab (anti-PD-L1 antibody) & 1 & NCT01938612 \\
\hline \multicolumn{4}{|l|}{ Dual ICB } \\
\hline & Nivolumab + ipilimumab (anti-CTLA-4 antibody) & $\|$ & NCT03101566 \\
\hline & Nivolumab + ipilimumab (anti-CTLA-4 antibody) & $\|$ & NCT02923934 \\
\hline & Nivolumab + ipilimumab (anti-CTLA-4 antibody) & $\|$ & NCT02834013 \\
\hline & Durvalumab + tremelimumab (anti-CTLA-4 antibody) & 1 & NCT01938612 \\
\hline \multicolumn{4}{|c|}{ ICB + immunomodulatory agent } \\
\hline & Pembrolizumab + GC-CSF & $\|$ & NCT02703714 \\
\hline & Pembrolizumab + Peg-IFNa-2b & $\|$ & NCT02982720 \\
\hline \multicolumn{4}{|c|}{ ICB + local treatment } \\
\hline & Tremelimumab + TACE, RFA, cryoablation or SBRT & 1 & NCT01853618 \\
\hline & Durvalumab + tremelimumab + TACE, RFA or cryoablation & II & NCT02821754 \\
\hline \multicolumn{4}{|l|}{ ICB + chemotherapy } \\
\hline & Pembrolizumab + mFOLFOX6 & 1 & NCT02268825 \\
\hline & Pembrolizumab + capecitabine/oxaliplatin & $\|$ & NCT03111732 \\
\hline & Nivolumab + gemcitabine/cisplatin & $\|$ & NCT03101566 \\
\hline & Durvalumab + tremelimumab + gemcitabine/cisplatin & $\|$ & NCT03046862 \\
\hline & SHR-1210 + gemcitabine/oxaliplatin & $\|$ & NCT03486678 \\
\hline & Durvalumab + guadecitabine & 1 & NCT03257761 \\
\hline \multicolumn{4}{|c|}{ ICB + molecularly targeted therapy } \\
\hline & Pembrolizumab + INCB054828 (FGFR inhibitor) & $|/| \mid$ & NCT02393248 \\
\hline & Pembrolizumab + XL888(HSP90 inhibitor) & 1 & NCT03095781 \\
\hline & Pembrolizumab + Ramucirumab (anti-VEGFR2 antibody) & 1 & NCT02443324 \\
\hline & Atezotizumab (anti-PD-L1 antibody) + Cobimetinib (MEK inhibitor) & $\|$ & NCT03201458 \\
\hline & Nivolumab + ABBV-368 & 1 & NCT03071757 \\
\hline \multicolumn{4}{|l|}{ ICB + adoptive cell } \\
\hline & Pembrolizumab + tumor infiltrating lymphocytes & II & NCT01174121 \\
\hline
\end{tabular}

ICB, immune checkpoint blockade

(NCT02628067). On the other hand, in a study in 86 patients with MMR-deficient tumors, including 4 CCAs, the ORR for pembrolizumab treatment was 53\% (74). One CCA patient (25\%) had a complete response and the other three showed stable disease (74). A number of clinical trials evaluating immunotherapy approaches are currently ongoing and these promising preliminary data warrant larger studies and bring significant effect for combination therapies to this intractable disease.

\section{SUMMARY AND FUTURE PERSPECTIVE}

With substantial understanding of the genetic aberrations in subtypes of CCA from recent studies, a precision medicine approach should be established and implemented in this largely intractable disease. The recently described molecular pathogenesis based on whole-gene sequence analysis in CCA needs further investigation in oncological function and in correlative studies in clinical trials. Therapies targeting FGFR2 and IDH1 have recently yielded promising initial results in iCCA, and ICB immunotherapy has also shown activity in stabilizing disease progression. Potential biomarkers for treatment selection have been identified and multiple clinical trials are ongoing to test this approach. Despite the relatively low prevalence of oncogenic drivers, such as FGFR, IDH, BRAF or EGFR mutations, when considered together, a meaningful subset can be considered actionable mutations to targeted therapy. A prior study estimated that about $60 \%$ patients may have actionable mutations for targeting therapy in CCA (18). However, the role of combination therapies, especially including those targeting specific targets in the tumor cells and the immune environment, remains to be established in future studies. These developments might bring renewed hope for a breakthrough in overcoming the dismal prognosis of CCA. 


\section{Conflicts of Interest}

D.G.D. receives research funding from Bayer, Exelixis and Bristol Meyer Squibb, and received consultant fees from Tilos, Bayer, and twoXAR.

\section{REFERENCES}

1. de Groen PC, Gores GJ, LaRusso NF, Gunderson LL, Nagorney DM. Biliary tract cancers. N Engl J Med 1999;341:1368-78.

2. Tyson GL, El-Serag HB. Risk factors for cholangiocarcinoma Hepatology 2011;54:173-84.

3. Rizvi S, Gores GJ. Pathogenesis, diagnosis, and management of cholangiocarcinoma. Gastroenterology 2013;145:1215-29.

4. Saha SK, Zhu AX, Fuchs CS, Brooks GA. Forty-Year Trends in Cholangiocarcinoma Incidence in the U.S.: Intrahepatic Disease on the Rise. Oncologist 2016;21:594-9.

5. Oliveira IS, Kilcoyne A, Everett JM, Mino-Kenudson M, Harisinghani MG, Ganesan K. Cholangiocarcinoma: classification, diagnosis, staging, imaging features, and management. Abdom Radiol (NY) 2017:42:1637-49.

6. Khuntikeo N, Chamadol N, Yongvanit P, Loilome W, Namwat N, Sithithaworn $\mathrm{P}$, et al. Cohort profile: cholangiocarcinoma screening and care program (CASCAP). BMC Cancer 2015;15:459.

7. Valle J, Wasan $H$, Palmer DH, Cunningham D, Anthoney A, Maraveyas $\mathrm{A}$, et al. Cisplatin plus gemcitabine versus gemcitabine for biliary tract cancer. N Engl J Med 2010;362:1273-81.

8. DeLeon TT, Ahn DH, Bogenberger JM, Anastasiadis PZ, Arora M, Ramanathan RK, et al. Novel targeted therapy strategies for biliary tract cancers and hepatocellular carcinoma. Future Oncol 2018; 14:553-566

9. Jusakul $A$, Cutcutache I, Yong $\mathrm{CH}$, Lim JQ, Huang MN, Padmanabhan N, et al. Whole-Genome and Epigenomic Landscapes of Etiologically Distinct Subtypes of Cholangiocarcinoma. Cancer Discov 2017;7:1116-1135.

10. Kayhanian H, Smyth EC, Braconi C. Emerging molecular targets and therapy for cholangiocarcinoma. World J Gastrointest Oncol 2017; 9:268-280.

11. Murakami Y, Kubo S, Tamori A, Itami S, Kawamura E, Iwaisako K, et al. Comprehensive analysis of transcriptome and metabolome analysis in Intrahepatic Cholangiocarcinoma and Hepatocellular Carcinoma. Sci Rep 2015;5:16294

12. Saha SK, Parachoniak CA, Ghanta KS, Fitamant J, Ross KN, Najem MS, et al. Mutant IDH inhibits HNF-4alpha to block hepatocyte differentiation and promote biliary cancer. Nature 2014:513:110-14.

13. Law LY. Dramatic response to trastuzumab and paclitaxel in a patient with human epidermal growth factor receptor 2-positive metastatic cholangiocarcinoma. J Clin Oncol 2012;30:e271-273.

14. Lombardi $P$, Marino D, Fenocchio E, Chilà G, Aglietta M, Leone F. Emerging molecular target antagonists for the treatment of biliary tract cancer. Expert Opin Emerg Drugs 2018;23:63-75

15. Nakamura H, Arai Y, Totoki Y, Shirota T, Elzawahry A, Kato M, et al. Genomic spectra of biliary tract cancer. Nat Genet. 2015;47:1003-10.

16. Borad MJ, Champion MD, Egan JB, Liang WS, Fonseca R, Bryce AH et al. Integrated genomic characterization reveals novel, therapeutically relevant drug targets in FGFR and EGFR pathways in sporadic intrahepatic cholangiocarcinoma. PLoS Genet 2014;10:e1004135.

17. Graham RP, Barr Fritcher EG, Pestova E, Schulz J, Sitailo LA Vasmatzis $\mathrm{G}$, et al. Fibroblast growth factor receptor 2 translocations in intrahepatic cholangiocarcinoma. Hum Pathol 2014;45:1630-38.

18. Ross JS, Wang K, Gay L, Al-Rohil R, Rand JV, Jones DM,et al. New routes to targeted therapy of intrahepatic cholangiocarcinomas revealed by next-generation sequencing. Oncologist 2014;19:235-42.

19. Wu YM, Su F, Kalyana-Sundaram S, Khazanov N, Ateeq B, Cao X, et al. Identification of targetable FGFR gene fusions in diverse cancers. Cancer Discov 2013;3:636-47.

20. Sia D, Losic B, Moeini A, Cabellos L, Hao K, Revill K, et al. Massive parallel sequencing uncovers actionable FGFR2-PPHLN1 fusion and
ARAF mutations in intrahepatic cholangiocarcinoma. Nat Commun 2015;6:6087.

21. Rizvi NA, Hellmann MD, Snyder A, Kvistborg P, Makarov V, Havel JJ, et al. Cancer immunology. Mutational landscape determines sensitivity to PD-1 blockade in non-small cell lung cancer. Science 2015;348:124-8.

22. Snyder A, Makarov V, Merghoub T, Yuan J, Zaretsky JM, Desrichard $A$, et al. Genetic basis for clinical response to CTLA-4 blockade in melanoma. N Engl J Med 2014;371:2189-99.

23. Sia D, Hoshida Y, Villanueva A, Roayaie S, Ferrer J, Tabak B, et al. Integrative molecular analysis of intrahepatic cholangiocarcinoma reveals 2 classes that have different outcomes. Gastroenterology 2013:144:829-40.

24. Andersen JB, Spee B, Blechacz BR, Avital I, Komuta M, Barbour A, et al. Genomic and genetic characterization of cholangiocarcinoma identifies therapeutic targets for tyrosine kinase inhibitors. Gastroenterology 2012;142:1021-31.e1015.

25. Bromberg JF, Wrzeszczynska MH, Devgan G, Zhao Y, Pestell RG, Albanese C, et al. Stat3 as an oncogene. Cell 1999;98:295-303.

26. Zhong W, Dai L, Liu J, Zhou S. Cholangiocarcinoma associated genes identified by integrative analysis of gene expression data. Mol Med Rep 2018;17:5744-53.

27. Oishi N, Kumar MR, Roessler S, Ji J, Forgues M, Budhu A, et al. Transcriptomic profiling reveals hepatic stem-like gene signatures and interplay of miR-200c and epithelial-mesenchymal transition in intrahepatic cholangiocarcinoma. Hepatology 2012;56:1792-1803.

28. Seol MA, Chu IS, Lee MJ, Yu GR, Cui XD, Cho BH, et al. Genomewide expression patterns associated with oncogenesis and sarcomatous transdifferentation of cholangiocarcinoma. BMC Cancer 2011;11:78.

29. Sulpice L, Rayar M, Desille M, Turlin B, Fautrel A, Boucher E, et al. Molecular profiling of stroma identifies osteopontin as an independent predictor of poor prognosis in intrahepatic cholangiocarcinoma. Hepatology 2013;58:1992-2000.

30. Hass HG, Nehls 0 , Jobst J, Frilling A, Vogel U, Kaiser S. Identification of osteopontin as the most consistently over-expressed gene in intrahepatic cholangiocarcinoma: detection by oligonucleotide microarray and real-time PCR analysis. World J Gastroenterol 2008; 14:2501-2510.

31. Sulpice L, Rayar M, Desille M, Turlin B, Fautrel A, Boucher E, et al. Molecular profiling of stroma identifies osteopontin as an independent predictor of poor prognosis in intrahepatic cholangiocarcinoma. Hepatology 2013;58:1992-2000.

32. Tongtawee T, Kaewpitoon SJ, Loyd R, Chanvitan S, Leelawat K, Praditpol N, et al. High Expression of Matrix Metalloproteinase-11 indicates Poor Prognosis in Human Cholangiocarcinoma. Asian Pac J Cancer Prev 2015;16:3697-701.

33. Huang QX, Cui JY, Ma H, Jia XM, Huang FL, Jiang LX. Screening of potential biomarkers for cholangiocarcinoma by integrated analysis of microarray data sets. Cancer Gene Ther 2016;23:48-53.

34. Sawanyawisuth K, Wongkham C, Araki N, Zhao Q, Riggins GJ, Wongkham S. Serial analysis of gene expression reveals promising therapeutic targets for liver fluke-associated cholangiocarcinoma. Asian Pac J Cancer Prev 2012;13 Suppl:89-93.

35. Giussani M, Merlino G, Cappelletti V, Tagliabue E, Daidone MG. Tumor-extracellular matrix interactions: Identification of tools associated with breast cancer progression. Semin Cancer Biol 2015; 35:3-10.

36. Razumilava N, Gores GJ. Cholangiocarcinoma. Lancet 2014;383: 2168-79.

37. Dang L, White DW, Gross S, Bennett BD, Bittinger MA, Driggers EM, et al. Cancer-associated IDH1 mutations produce 2-hydroxyglutarate. Nature 2009;462:739-44.

38. Losman JA, Kaelin WG. What a difference a hydroxyl makes: mutant IDH, (R)-2-hydroxyglutarate, and cancer. Genes Dev 2013;27:836-52.

39. Lu C, Ward PS, Kapoor GS, Rohle D, Turcan S, Abdel-Wahab O, et al. IDH mutation impairs histone demethylation and results in a block to cell differentiation. Nature 2012:483:474-78.

40. Sasaki M, Knobbe CB, Munger JC, Lind EF, Brenner D, Brüstle A, et al. IDH1(R132H) mutation increases murine haematopoietic progenitors and alters epigenetics. Nature 2012;488:656-59. 
41. Xu W, Yang H, Liu Y, Yang Y, Wang P, Kim SH, et al. Oncometabolite 2-hydroxyglutarate is a competitive inhibitor of $\alpha$-ketoglutaratedependent dioxygenases. Cancer Cell 2011;19:17-30.

42. Farshidfar F, Zheng S, Gingras MC, Newton Y, Shih J, Robertson AG, et al. Integrative Genomic Analysis of Cholangiocarcinoma Identifies Distinct IDH-Mutant Molecular Profiles. Cell Rep 2017;19:2878-80.

43. Lee H, Ross JS. The potential role of comprehensive genomic profiling to guide targeted therapy for patients with biliary cancer. Therap Adv Gastroenterol 2017;10:507-20.

44. Churi CR, Shroff R, Wang Y, Rashid A, Kang HC, Weatherly J, et al. Mutation profiling in cholangiocarcinoma: prognostic and therapeutic implications. PLoS One 2014;9:e115383.

45. Rohle D, Popovici-Muller J, Palaskas N, Turcan S, Grommes C, Campos C, et al. An inhibitor of mutant IDH1 delays growth and promotes differentiation of glioma cells. Science 2013;340:626-30.

46. Wang F, Travins J, DeLaBarre B, Penard-Lacronique V, Schalm S Hansen E, et al. Targeted inhibition of mutant IDH2 in leukemia cells induces cellular differentiation. Science 2013;340:622-26.

47. Amatangelo MD, Quek L, Shih A, Stein EM, Roshal M, David MD, et al. Enasidenib induces acute myeloid leukemia cell differentiation to promote clinical response. Blood 2017;130:732-41.

48. Kats LM, Vervoort SJ, Cole R, Rogers AJ, Gregory GP, Vidacs E, et al. A pharmacogenomic approach validates AG-221 as an effective and on-target therapy in IDH2 mutant AML. Leukemia 2017;31: 1466-70.

49. Thomas D, Majeti R. Optimizing Next-Generation AML Therapy: Activity of Mutant IDH2 Inhibitor AG-221 in Preclinical Models. Cancer Discov 2017;7:459-461.

50. Rizvi S, Yamada D, Hirsova P, Bronk SF, Werneburg NW, Krishnan A et al. A Hippo and Fibroblast Growth Factor Receptor Autocrine Pathway in Cholangiocarcinoma. J Biol Chem 2016;291:8031-47.

51. Javle M, Lowery M, Shroff RT, Weiss KH, Springfeld C, Borad MJ, Ramanathan RK, et al. Phase II Study of BGJ398 in Patients With FGFR-Altered Advanced Cholangiocarcinoma. J Clin Oncol 2018;36:276-282.

52. Perera TPS, Jovcheva E, Mevellec L, Vialard J, De Lange D, Verhulst $\mathrm{T}$, et al. Discovery and Pharmacological Characterization of JNJ42756493 (Erdafitinib), a Functionally Selective Small-Molecule FGFR Family Inhibitor. Mol Cancer Ther 2017;16:1010-1020.

53. Tabernero J, Bahleda R, Dienstmann R, Infante JR, Mita A, Italiano A, et al. Phase I Dose-Escalation Study of JNJ-42756493, an Oral Pan-Fibroblast Growth Factor Receptor Inhibitor, in Patients With Advanced Solid Tumors. J Clin Oncol 2015;33:3401-08.

54. Zhu AX, Borger DR, Kim Y, Cosgrove D, Ejaz A, Alexandrescu S, et al. Genomic profiling of intrahepatic cholangiocarcinoma: refining prognosis and identifying therapeutic targets. Ann Surg Oncol 2014; 21:3827-34

55. Bekaii-Saab T, Phelps MA, Li X, Saji M, Goff L, Kauh JS, et al. Multiinstitutional phase II study of selumetinib in patients with metastatic biliary cancers. J Clin Oncol 2011:29:2357-63.

56. Bridgewater J, Lopes A, Beare S, Duggan M, Lee D, Ricamara M, et al. A phase 1b study of Selumetinib in combination with Cisplatin and Gemcitabine in advanced or metastatic biliary tract cancer: the ABC-04 study. BMC Cancer 2016:16:153.

57. Goeppert B, Frauenschuh L, Renner M, Roessler S, Stenzinger A Klauschen $F$, et al. BRAF V600E-specific immunohistochemistry reveals low mutation rates in biliary tract cancer and restriction to intrahepatic cholangiocarcinoma. Mod Pathol 2014;27: 1028-34.

58. Hyman DM, Puzanov I, Subbiah V, Faris JE, Chau I, Blay JY, et al.
Vemurafenib in Multiple Nonmelanoma Cancers with BRAF V600 Mutations. N Engl J Med 2015;373:726-36.

59. Miyamoto M, Ojima H, Iwasaki M, Shimizu H, Kokubu A, Hiraoka N, et al. Prognostic significance of overexpression of c-Met oncoprotein in cholangiocarcinoma. Br J Cancer 2011;105:131-38.

60. Nakazawa K, Dobashi $Y$, Suzuki S, Fujii H, Takeda $Y$, Ooi A. Amplification and overexpression of c-erbB-2, epidermal growth factor receptor, and c-met in biliary tract cancers. J Pathol 2005; 206:356-65

61. Aishima SI, Taguchi KI, Sugimachi K, Shimada M, Tsuneyoshi M. cerbB-2 and c-Met expression relates to cholangiocarcinogenesis and progression of intrahepatic cholangiocarcinoma. Histopathology 2002:40:269-78.

62. Pant S, Saleh M, Bendell J, Infante JR, Jones S, Kurkjian CD, et al. A phase I dose escalation study of oral c-MET inhibitor tivantinib (ARQ 197) in combination with gemcitabine in patients with solid tumors. Ann Oncol 2014;25:1416-21.

63. Goyal L, Zheng H, Yurgelun MB, Abrams TA, Allen JN, Cleary JM, et al. A phase 2 and biomarker study of cabozantinib in patients with advanced cholangiocarcinoma. Cancer 2017; 123:1979-88.

64. Pignochino Y, Sarotto I, Peraldo-Neia C, Penachioni JY, Cavalloni G, Migliardi G, et al. Targeting EGFR/HER2 pathways enhances the antiproliferative effect of gemcitabine in biliary tract and gallbladder carcinomas. BMC Cancer 2010:10:631.

65. El-Khoueiry AB, Rankin C, Siegel AB, lqbal S, Gong IY, Micetich KC, et al. S0941: a phase 2 SWOG study of sorafenib and erlotinib in patients with advanced gallbladder carcinoma or cholangiocarcinoma. Br J Cancer 2014; 110:882-87.

66. Shi RY, Yang XR, Shen QJ, Yang LX, Xu Y, Qiu SJ, et al. High expression of Dickkopf-related protein 1 is related to lymphatic metastasis and indicates poor prognosis in intrahepatic cholangiocarcinoma patients after surgery. Cancer 2013;119:993-1003.

67. Chae WJ, Ehrlich AK, Chan PY, Teixeira AM, Henegariu O, Hao L, et al. The Wnt Antagonist Dickkopf-1 Promotes Pathological Type 2 Cell-Mediated Inflammation. Immunity 2016;44:246-58.

68. D'Amico L, Mahajan S, Capietto AH, Yang Z, Zamani A, Ricci B, Bumpass DB, et al. Dickkopf-related protein 1 (Dkk1) regulates the accumulation and function of myeloid derived suppressor cells in cancer. J Exp Med 2016;213:827-840.

69. Malladi S, Macalinao DG, Jin X, He L, Basnet H, Zou Y, et al. Metastatic Latency and Immune Evasion through Autocrine Inhibition of WNT. Cell 2016;165:45-60.

70. Gani F, Nagarajan N, Kim Y, Zhu Q, Luan L, Bhaijjee F, et al. Program Death 1 Immune Checkpoint and Tumor Microenvironment: Implications for Patients With Intrahepatic Cholangiocarcinoma. Ann Surg Oncol 2016;23:2610-17.

71. Fontugne J, Augustin J, Pujals A, Compagnon P, Rousseau B, Luciani A, et al. PD-L1 expression in perihilar and intrahepatic cholangiocarcinoma. Oncotarget 2017;8:24644-651.

72. Naboush A, Roman CA, Shapira I. Immune checkpoint inhibitors in malignancies with mismatch repair deficiency: a review of the state of the current knowledge. J Investig Med 2017;65:754-58.

73. Schumacher TN, Schreiber RD. Neoantigens in cancer immunotherapy. Science 2015;348:69-74.

74. Le DT, Durham JN, Smith KN, Wang H, Bartlett BR, Aulakh LK, et al. Mismatch repair deficiency predicts response of solid tumors to PD-1 blockade. Science 2017:357:409-13.

75. Silva VW, Askan G, Daniel TD, Lowery M, Klimstra DS, Abou-Alfa GK, et al. Biliary carcinomas: pathology and the role of DNA mismatch repair deficiency. Chin Clin Oncol 2016;5:62. 